\title{
Suppression of $\alpha$-MSH and IBMX-induced melanogenesis by cordycepin via inhibition of CREB and MITF, and activation of PI3K/Akt and ERK-dependent mechanisms
}

\author{
MEI LING JIN ${ }^{4 *}$, SUN YOUNG PARK ${ }^{1 *}$, YOUNG HUN KIM ${ }^{1}$, GEUNTAE PARK $^{2}$, \\ HONG-JOO SON ${ }^{3}$ and SANG-JOON LEE ${ }^{4}$
}

\begin{abstract}
${ }^{1}$ Bio-IT Fusion Technology Research Institute; ${ }^{2}$ Institute for Research and Industry Cooperation; ${ }^{3}$ Department of Life Science and Environmental Biochemistry; ${ }^{4}$ Department of Microbiology, Pusan National University, Busan, Republic of Korea
\end{abstract}

Received July 25, 2011; Accepted September 9, 2011

DOI: $10.3892 / \mathrm{ijmm} .2011 .807$

\begin{abstract}
Cordycepin has been a traditional medicine in China and Korea for centuries. This study explored the inhibitory effect of cordycepin on melanogenesis and the relative molecular mechanisms. Cordycepin inhibited melanin synthesis-related enzymes, such as tyrosinase, tyrosinaserelated protein-1 (TRP1) and tyrosinase-related protein-2 (TRP2). $\alpha$-MSH and IBMX were reported as melanin synthesis enhancers. Both of them could increase intracellular melanin synthesis by activation of the microphthalmia-associated transcription factor (MITF) signaling pathway. In the MITF pathway, the phosphorylation of cAMP related binding protein (CREB) activated the transcription of MITF, resulting in increasing melanin synthesis. Cordycepin also decreased the phosphorylation of CREB induced by $\alpha-\mathrm{MSH}$ and IBMX in B16F10 melanoma cells. Accordingly, cordycepin inhibited melanogenesis signaling pathways by activating ERK and AKT signaling pathways to regulate the suppression of MITF and its downstream pathways including tyrosinase, TRP1 and TRP2. These results indicate the role of cordycepin as a potent depigmenting agent for cosmetics.
\end{abstract}

\section{Introduction}

Melanin, which is synthesized in intracellular organelles designated melanosomes, is a major pigment of skin color. Melanogenesis is regulated by three specific enzymes: tyrosinase, tyrosinase-related protein-1 (TRP-1), and tyrosinaserelated protein-2 (TRP-2). Tyrosinase, a copper-containing

Correspondence to: Dr Sang-Joon Lee, Department of Microbiology, Pusan National University, Busan 609-735, Republic of Korea

E-mail: sangjoon@pusan.ac.kr

*Contributed equally

Key words: cordycepin, melanin, tyrosinase, cAMP-responsive element binding protein, microphthalmia-associated transcription factor, PI3K/Akt, extracellularly responsive kinases glycoprotein, is a key enzyme in melanin synthesis and a ratelimiting enzyme in this pathway, and can catalyze three different reactions: the hydroxylation of tyrosine to 3, 4-dihydroxyphenylalanine (DOPA), the oxidation of DOPA to dopaquinone and the conversion of dopaquinone to dopachrome, and then to dihydro-indolizine (DHI) or indole 5,6-quinone-2-carboxylic acid (DHICA) (1-5). During this biosynthesis pathway, TRP-1 and TRP-2 have functional roles. TRP-1 catalyzes the oxidation of DHICA, and TRP-2 (dopachrome tautomerase) catalyzes the conversion of dopachrome to DHICA (6). In addition, the two enzymes are regulated by a specific transcription factor, microphthalmia-associated transcription factor (MITF) (7-9).

MITF is regulated by several signaling pathways. Mitogenactivated protein kinases (MAPKs) are a family of serine/ threonine kinases that regulate cell differentiation, proliferation, and cellular activities. The MAPK superfamily contains three well characterized subfamilies: the extracellularly responsive kinases (also termed extracellular signal-regulated kinases; ERKs), the c-Jun N-terminal kinases (JNKs), and the p38-MAPKs. MAPKs play essential roles in melanogenesis $(10,11)$. Moreover, investigations suggest that the p38 MAPK pathway activation has been reported to be related to increased melanin synthesis $(12,13)$, and the activation of ERK signaling down-regulates melanogenesis by inhibiting the tyrosinase activity (14). In addition, the activation of Akt signaling also plays a key role in inhibiting melanogenesis. These observations support the suggestion that the inhibitory effect of natural compounds on melanogenesis may be dependent on control of the upstream signaling pathways related to the activation and expression of tyrosinase (14).

Melanogenesis can be induced by several extracellular stimuli, such as ultraviolet (UV) light, chemicals including $\alpha$-melanocyte-stimulating hormone $(\alpha-\mathrm{MSH})$ and isobutylmethylxanthine (IBMX). $\alpha$-MSH combined with its receptor MC1R induces the enhanced expression of MITF, dependent on increased cyclic-AMP (cAMP) levels, leading to increased expression of tyrosinase and other melanogenesis-related enzymes. The end result is increased melanin synthesis. This pathway is apparently regulated by activation of ERK, whose phosphorylation allows the expression of MITF, leading to its degradation. On the other hand, the activation of ERK can 
result in phosphorylation of cAMP related binding protein (CREB), which binds to the CRE consensus motif in the MITF promoter to up-regulate MITF gene expression (15).

Numerous chemicals and cosmetic agents inhibit melanin biosynthesis by inhibiting tyrosinase catalytic activity. Many skin whiting agents including kojic acid, linoleic acid, hinokitol, and arbutin possess anti-melanogenesis activity. Arbutin is a $\beta$-D-glucopyranoside derivative of hydroquinone that inhibits melanin synthesis at a certain concentration (16).However, some of the other agents can produce side effects and cellular toxicity. To avoid those negative aspects, natural compounds derived from plant or fungi extract have been investigated. Many extracts from plants and fungi extraction inhibit melanogenesis and have been incorporated into cosmetic preparations. For example, the Hoelen extract inhibits melanogensis and hyperpigmentation in skin cells (17). Other anti-melanogenesis compounds include lucidone extract from Lindera erythrocapa (18), palmitoleic acid (19), and the hirseins extract from Thymelaea hirsute (20).

Cordycepin is extracted from fungi of genus Cordyceps, and has been used traditionally as an anti-tumor, anti-viral, anti-inflammatory agent in East Asian countries (21-26). In this study, we examined the inhibitory effect of cordycepin on the melanogenesis signaling pathways, including the expression of MITF, tyrosinase, TRP1 and TRP2, and the phosphorylation of CREB, via the activation of ERK and Akt in murine B16F10 melanoma cells stimulated by $\alpha-\mathrm{MSH}$ and IBMX.

\section{Materials and methods}

Materials. Cordycepin, $\alpha$-MSH, IBMX, L-3, 4-dihydroxyphenylalanine (L-DOPA), arbutin, and all other reagents were purchased from Sigma-Aldrich. Antibodies recognizing phospho-CREB and CREB were obtained from Cell Signaling Technology (Danvers, MA, USA). Tyrosinase, TRP1, TRP2 and MITF antibodies were supplied by Santa Cruz Biotechnology (Santa Cruz, CA, USA).

Cell culture. Cells of the B16F10 murine melanoma cell line obtained from the American Type Culture Collection (Rockville, MD, USA) were grown as monolayers in Dulbecco's modified Eagle's medium (DMEM; Gibco-BRL, Carlsbad, CA, USA) supplemented with $10 \%$ heat-inactivated fetal bovine serum (FBS; Gibco-BRL). The cells were incubated at $37^{\circ} \mathrm{C}$ in a humidified atmosphere composed of $5 \%$ $\mathrm{CO}_{2}$ and $95 \%$ air. To avoid changes in cell characteristics produced by extended cell culture periods, cells were used between passages 15 and 25. Each cell suspension was split every 2 days to maintain exponential growth.

Cell viability assay. The cells were incubated in wells of a 24-well plate at a density of $4 \times 10^{4}$ cells/well. 3-(4,5-Dimethylthiazol-2-yl)-2,5-diphenyltetrazolium bromide (MTT; $50 \mu \mathrm{g} / \mathrm{ml}$ ) was added to each well. The plates were incubated for $3 \mathrm{~h}$ at $37^{\circ} \mathrm{C}$ in a $5 \% \mathrm{CO}_{2}$ atmosphere, after which the supernatant was removed and the formazan crystals that had formed in the viable cells were solubilized with dimethylsulfoxide (DMSO). The absorbance of each well was then read at $570 \mathrm{~nm}$ using an enzyme-linked immunosorbent assay (ELISA) reader (Wallace, Boston, MA, USA).
Determination of extracellular melanin content. Cells were treated with $1 \mu \mathrm{M} \alpha-\mathrm{MSH}$ in the absence or presence of cordycepin for $48 \mathrm{~h}$. The culture medium was harvested and centrifuged at $10,000 \mathrm{rpm}$ for $10 \mathrm{~min}$. The relative melanin content was determined by measuring the absorbance at $405 \mathrm{~nm}$ in a microplate reader. Melanin production was calculated by normalizing the melanin values with protein content (absorbance $/ \mu \mathrm{g}$ protein). For this purpose, protein content was determined using the Bradford dye reagent (Bio-Rad, Hercules, CA, USA).

Determination of intracellular melanin content. Cells were treated with $1 \mu \mathrm{M} \alpha-\mathrm{MSH}$ in the absence or presence of cordycepin for $48 \mathrm{~h}$. The cell pellets were then dissolved in $500 \mu \mathrm{l}$ of $1 \mathrm{~N} \mathrm{NaOH}$ in $10 \% \mathrm{DMSO}$ at $80^{\circ} \mathrm{C}$ for $1 \mathrm{~h}$. The relative melanin content was determined by measuring the absorbance at $475 \mathrm{~nm}$ in a microplate reader. Melanin production was calculated as described above.

B16F10 cell tyrosinase activity assay. Tyrosinase activity was determined by measuring the rate of dopachrome formation of L-DOPA. Cells grown in wells of 6-well dishes were treated with $1 \mu \mathrm{M} \alpha-\mathrm{MSH}$ in the absence or presence of cordycepin for $48 \mathrm{~h}$. The cells were then washed in ice-cold phosphatebuffered saline (PBS) and lysed in PBS containing 1\% (w/v) Triton X-100. The tyrosinase substrate L-DOPA $(2 \mathrm{mg} / \mathrm{ml})$ was prepared in the same phosphate lysis buffer. Each extract was placed in wells of a 96-well plate and the enzymatic assay was commenced by adding L-DOPA solution. After incubation, dopachrome formation was assayed by measuring absorbance at $475 \mathrm{~nm}$ using a microplate reader. The value of each measurement was expressed as a percentage of the control.

Quantitative real-time polymerase chain reaction (PCR). Total-RNA was isolated from cells using the RNA Mini Spin RNA isolation kit (GE Healthcare, Buckinghamshire, UK) according to the manufacturer's instructions. cDNA was synthesized from $1 \mu \mathrm{g}$ of total-RNA using Maxime RT PreMix (Intron Biotechnology, Seongnam, Korea) and anchored oligo(dt)15-primers. Real-time PCR was performed with a Chromo4 $^{\mathrm{TM}}$ instrument (Bio-Rad) using the SYBR-Green master mix (Applied Biosystems, Foster City, CA, USA). The relative amount of target mRNA was determined using an established comparative threshold $(\mathrm{Ct})$ method by normalizing target mRNA Ct values to those for glyceraldehyde 3-phosphate dehydrogenase $(\Delta \mathrm{Ct})$.

Western blot analysis. Cells were harvested in ice-cold lysis buffer consisting of $1 \%$ Triton X-100, 1\% deoxycholate, and $0.1 \%$ sodium dodecyl sulfate (SDS). The protein content of the cell lysates was then determined using the Bradford reagent (Bio-Rad). The protein in each sample (50 $\mu \mathrm{g}$ total protein) was resolved by $10 \%$ SDS-polyacrylamide gel electrophoresis (SDS-PAGE), transferred to a polyvinylidene difluoride (PVDF) membrane and exposed to the appropriate antibody. The proteins were visualized by an enhanced chemiluminescence detection system (Amersham Biosciences, Piscataway, NJ, USA) using horseradish peroxidase-conjugated anti-rabbit or anti-mouse secondary antibody. Images were acquired 
A
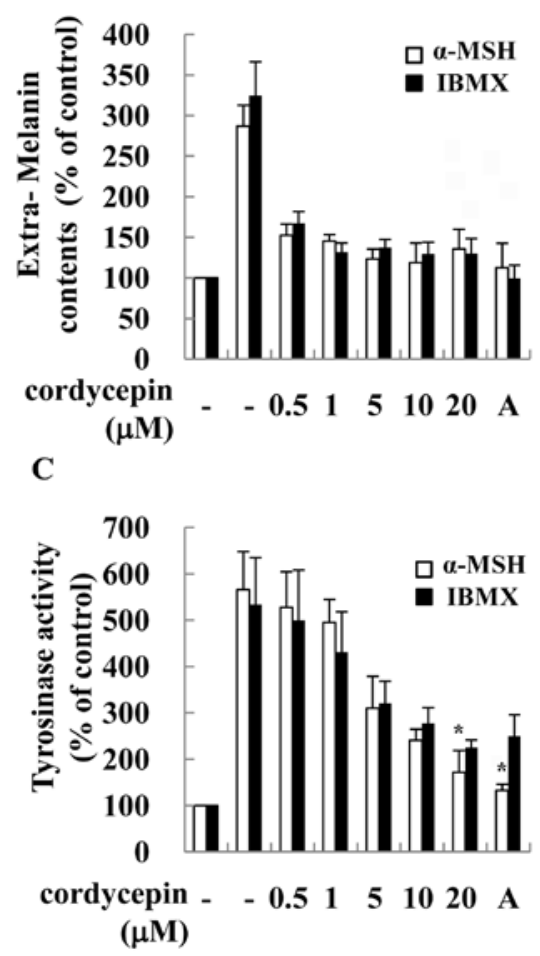

B
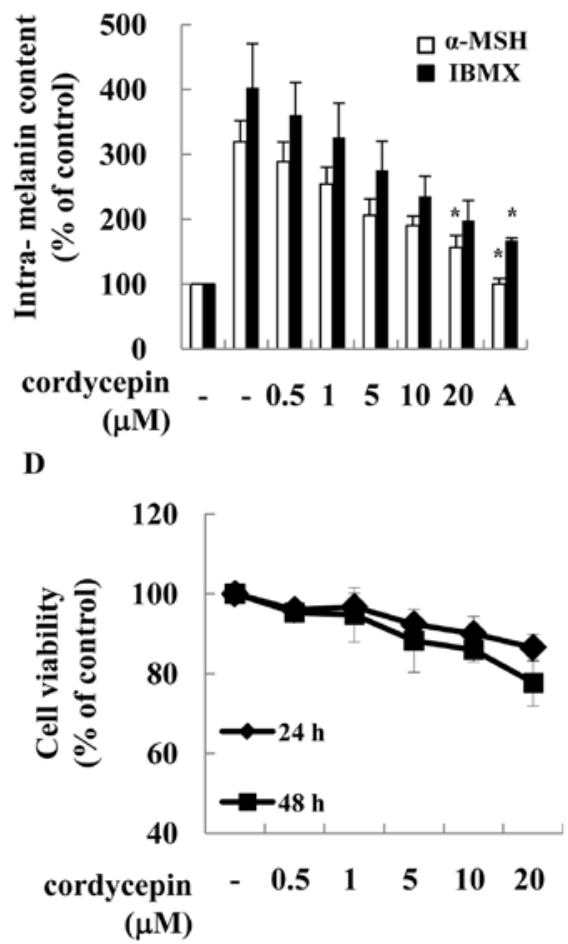

Figure 1. Effect of cordycepin on $\alpha$-MSH- and IBMX-induced melanin synthesis and tyrosinase activity in B16F10 cells. Cells were incubated with various concentrations of cordycepin or arbutin $(200 \mu \mathrm{M})$ for $2 \mathrm{~h}$, and then stimulated with $0.5 \mu \mathrm{M}$ of $\alpha$-MSH or $0.1 \mathrm{mM}$ of IBMX for $48 \mathrm{~h}$. (A) Results of extracellularmelanin content assay. (B) Results of intracelluar-melanin content assay. (C) Tyrosinase activity assay was determined and melanin was calculated by adding the extracellular and intracellular mean values after normalization for protein content. (D) Cell viability was determined using an MTT assay. Values are mean \pm SE of three independent experiments. ${ }^{*} \mathrm{P}<0.05$ compared with the cells treated only with $\alpha-\mathrm{MSH}$ or IBMX.

using an ImageQuant 350 analyzer (Amersham Biosciences) and densitometry was performed using ImageQuant TL software (Amersham Biosciences).

Statistical analyses. The data are expressed as mean \pm standard error (SE). Each experiment was repeated at least three times. Statistical analysis was performed with SPSS version 16.0 software (SPSS, Chicago, IL, USA) to determine significant differences. One- or two-way ANOVA was followed by the Dunn's post-hoc test for analyses. Values of $\mathrm{P}<0.05$ were considered statistically significant.

\section{Results}

Cordycepin inhibits $\alpha$-MSH- and IBMX-induced cellular melanin synthesis and tyrosinase activity. Prior to investigating the pharmacological potential of cordycepin on $\alpha$-MSH- and IBMX-induced melanogenesis activity, the dose and time dependence of the cytotoxic effects of $0.5-20 \mu \mathrm{M}$ cordycepin in B16F10 cells were assessed using the MTT viability assay. The different concentrations of cordycepin that were used were not cytotoxic (Fig. 1D). The effect of cordycepin on $\alpha-\mathrm{MSH}$ - and IBMX-stimulated melanin synthesis is summarized in Fig. 1A and B. While both $\alpha-\mathrm{MSH}$ and IBMX are crucial cAMP-elevating agents, they act differently. $\alpha$-MSH combines the melanocortin 1 receptor (MC1R) and activates adenylate cyclase, which can raise the intracellular cAMP concentration. IBMX increases the intracellular
cAMP concentration by inhibiting cAMP phosphodiesterase. Presently, 5-20 $\mu \mathrm{M}$ cordycepin inhibited melanin synthesis induced by both $\alpha$-MSH and IBMX (Fig. 1A and B). The efficacious concentrations of cordycepin are much lower than the effective level $(200 \mu \mathrm{M})$ of arbutin, an established effective melanogenesis inhibitor and popular cosmetic agent. Cordycepin also decreased the melanin synthesis relative to protein tyrosinase activity (Fig. 1C).

Effect of cordycepin on the expression of tyrosinase, TRP1, and TRP2 in $\alpha$-MSH-induced B16F10 cells. To evaluate the molecular mechanism(s) of cordycepin, the influence of the compound on the production of tyrosinase, TRP1, and TRP2 were evaluated; production of all three proteins, especially tyrosinase and TRP1, was reduced by cordycepin (Fig. 2A). The mRNA levels of tyrosinase, TRP1, and TRP2 (Fig. 2B) were consistent with the production of their respective proteins, and were also dose-dependently inhibited by cordycepin.

Effect of cordycepin on the expression of MITF and phosphorylation of $C R E B$. MITF is a specific transcriptional factor that has a crucial role in melanogenesis as the major regulator of tyrosinase, TRP1, and TRP2 expression; down-regulation of MITF results in hypopigmentation. The expression of MITF can be regulated both transcriptionally and translationally. Cordycepin inhibited $\alpha$-MSH- and IBMX-induced melanin synthesis by a decrease in MITF expression and phosphorylation of CREB (Fig. 3). These results are consistent with the 
A
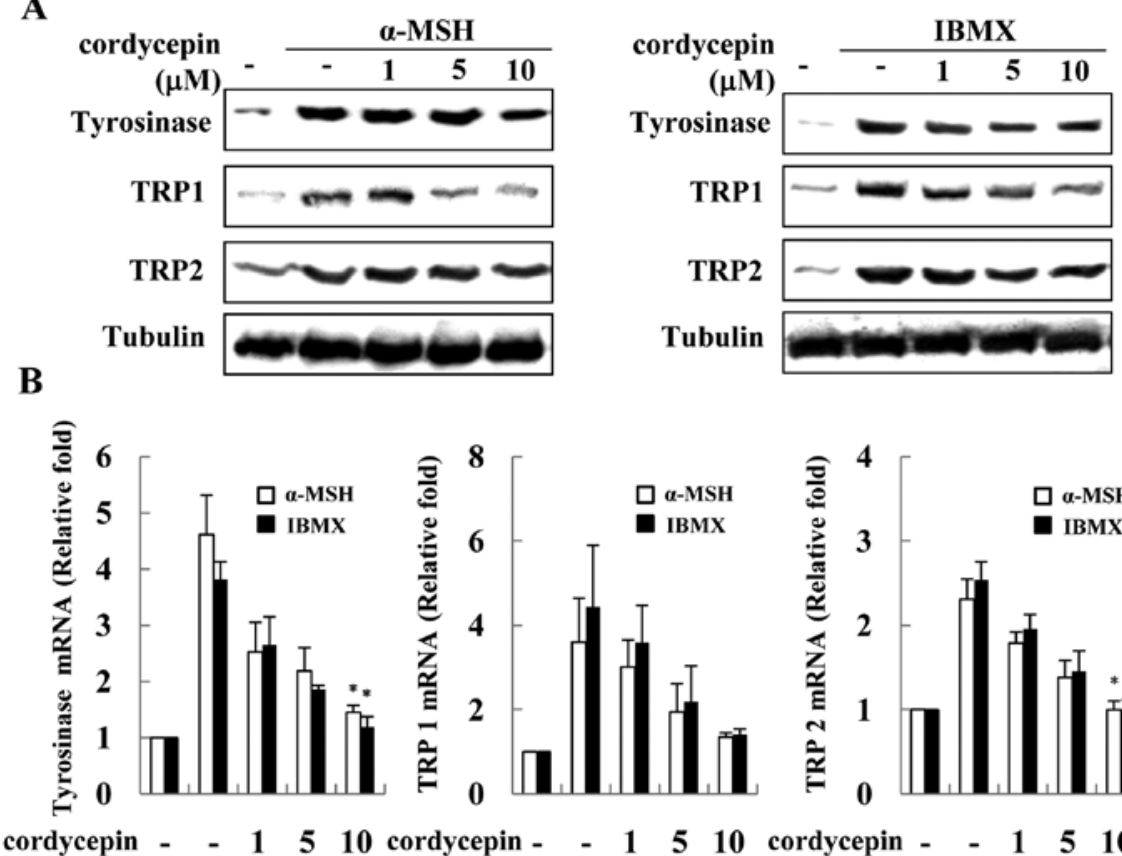

$(\mu \mathrm{M})$
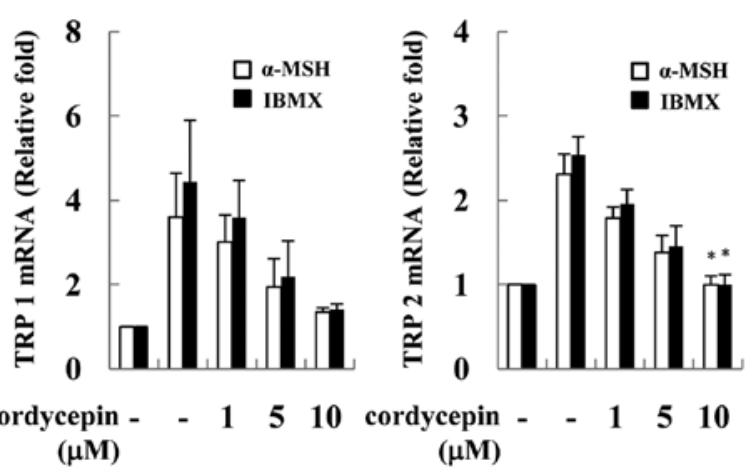

Figure 2. Effect of cordycepin on the expression of tyrosinase, TRP-1 and TRP-2 in $\alpha-\mathrm{MSH}$ - and IBMX-induced B16F10 cells. (A) Cells were incubated with the indicated cordycepin concentrations for $2 \mathrm{~h}$, and then exposed to $0.5 \mu \mathrm{M}$ of $\alpha-\mathrm{MSH}$ or $0.1 \mathrm{mM}$ of IBMX for $48 \mathrm{~h}$. Tyrosinase, TRP-1 and TRP-2 protein expression was decreased as indicated by Western blotting examination. Detection of $\alpha$-tubulin was used as a protein loading control for each lane. (B) Cells were pre-treated with cordycepin for $2 \mathrm{~h}$, and then exposed to $0.5 \mu \mathrm{M}$ of $\alpha$-MSH or $0.1 \mathrm{mM}$ of IBMX for $24 \mathrm{~h}$. mRNA levels of tyrosinase, TRP-1 and TRP-2 were examined by real-time PCR. Relative tyrosinase, TRP-1 and TRP-2 mRNA expression $\left(2^{-\Delta C}\right)$ was determined by real-time PCR and calculated

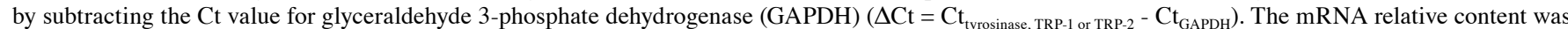
indicated as fold-change over control. Values are mean \pm SE of three independent experiments. "P $\mathrm{P}<0.05$ compared with the cells treated only with $\alpha$-MSH or IBMX.

A
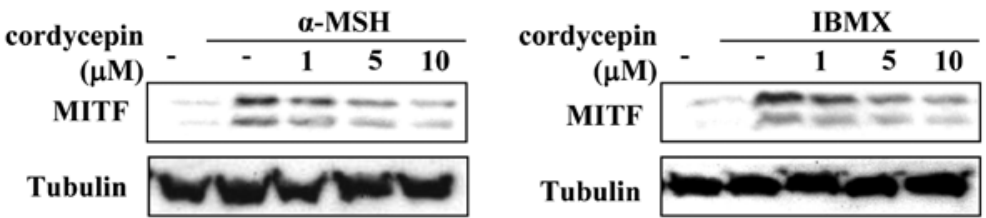

B
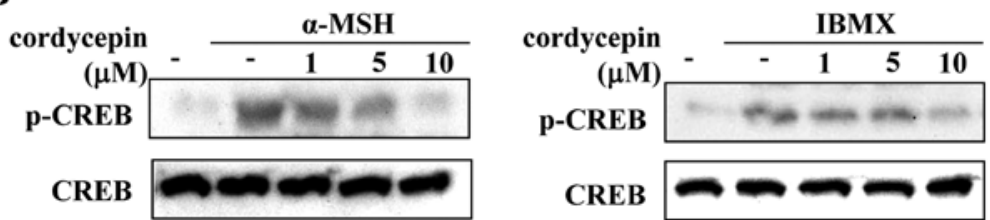

Figure 3. Effect of cordycepin on the expression of MITF in $\alpha-\mathrm{MSH}$ - or IBMX-induced B16F10 cells. (A) Cells were incubated with various concentrations of cordycepin for $2 \mathrm{~h}$, and then stimulated with $0.5 \mu \mathrm{M}$ of $\alpha$-MSH or $0.1 \mathrm{mM}$ of IBMX for $48 \mathrm{~h}$. MITF protein expression decreased as indicated by Western blotting. $\alpha$-tubulin was used as a protein loading control for each lane. (B) Cells were incubated with various concentrations of cordycepin for $2 \mathrm{~h}$, and then stimulated with $0.5 \mu \mathrm{M}$ of $\alpha$-MSH or $0.1 \mathrm{mM}$ of IBMX for $0.5 \mathrm{~h}$. The expression levels of phospho-CREB and CREB were examined by Western blotting. Equal protein loading was confirmed by $\alpha$-tubulin expression.

ability of cordycepin to block $\alpha$-MSH-enhanced MITF expression, suggesting that cordycepin suppresses $\alpha$-MSH-enhanced melanogenesis at the transcriptional level via the activation of CREB.

Effect of cordycepin on cellular melanin synthesis and tyrosinase activity in LY294002- or PD98059-treated B16F10 cells. The present study also investigated the dependence of the inhibitory effect of cordycepin on the ERK and AKT signaling pathways (Fig. 4). Activation of ERK by Scf/c-kit (a transmembrane receptor type tyrosine kinase) modulates $\alpha$-MSH-induced melanogenesis, and phosphorylation of ERK decreases expressed MITF and involves the phosphorylation of MITF. In order to determine the molecular mechanism of cordycepin on melanogenesis in B16 melanoma cells, we analyzed the pattern of MAPK phosphorylation in B16F10 
A

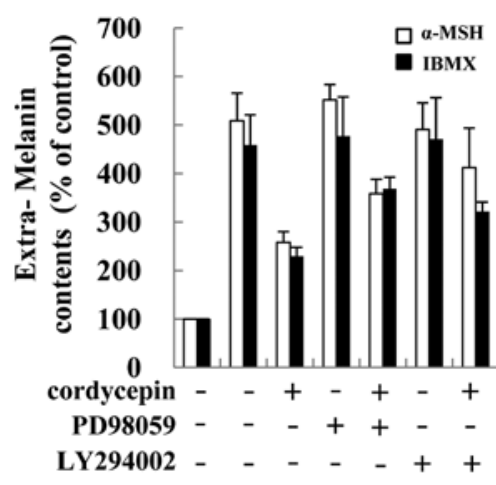

C

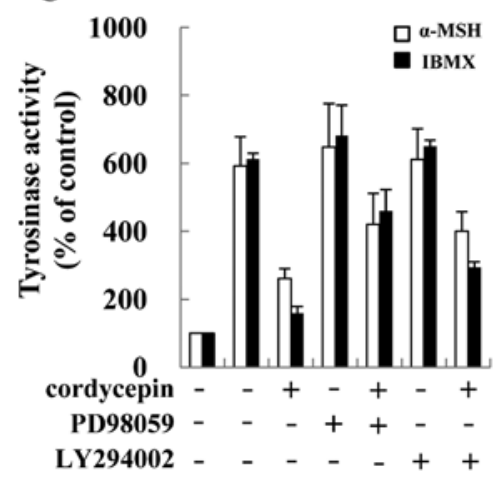

B

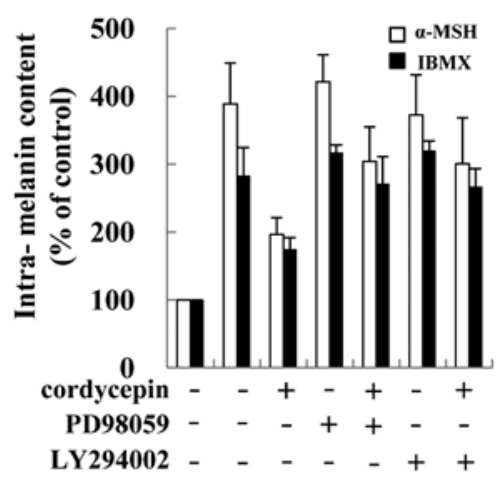

D

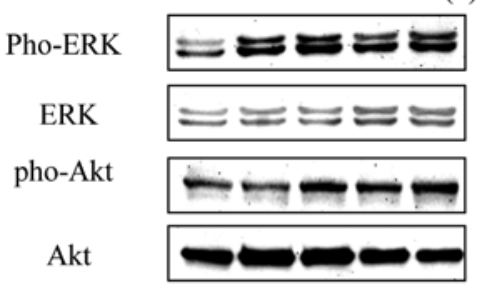

Figure 4. Effect of cordycepin on melanin synthesis and tyrosinase activity in PD98059- or LY294002-treated B16F10 cells. Cells were incubated with cordycepin $(20 \mu \mathrm{M})$ for $2 \mathrm{~h}$, and then stimulated with $0.5 \mu \mathrm{M}$ of $\alpha$-MSH and $0.1 \mathrm{mM}$ of IBMX in the absence and presence of PD98059 (20 $\mu \mathrm{M})$ or LY294002 $(20 \mu \mathrm{M})$ for $48 \mathrm{~h}$. (A) Extra-melanin content assay. (B) Intra-melanin content assay. (C) Tyrosinase activity assay. (D) Cells were treated with $20 \mu \mathrm{M}$ cordycepin for the indicated times and were subjected to Western blotting for Akt, phosphorylated Akt, ERK and phosphorylated ERK.

melanoma cells treated with cordycepin. The results shown in Fig. 4D, indicate that the levels of p-ERK and p-Akt were increased. To further confirm the role of ERK and Akt signaling pathways in cordycepin induced inhibition of melanogenesis, cells were co-treated with ERK (PD98059) or Akt (LY294002) inhibitors (Fig. 4). Cells treated with $\alpha$-MSH or IBMX, and PD98059 displayed increased activities of the melanogenesisrelated enzymes tyrosinase, TRP1, and TRP2 compared to cells treated only with $\alpha$-MSH or IBMX. The same pattern was evident using LY294002.

\section{Discussion}

Cordycepin has been a traditional medicine in East Asia for centuries. More recently, the ability of cordycepin to inhibit polyadenylation during $\alpha$-MSH induction of tyrosinase was reported (27). Previous studies have supported the potential of cordycepin as a melanogenesis inhibitor agent. In this study, we explored the possible effects of cordycepin on melanin synthesis. Evidently, this inhibition was correlated with down-regulation of MITF expression and the anti-whitening mechanism was mediated through cordycepin's inhibitory effect on ERK and Akt pathway-mediated signaling.

Firstly, we investigated the dose and time dependence of the cytotoxic effects of $0.5-20 \mu \mathrm{M}$ cordycepin in B16F10 cells by the MTT viability assay. These concentrations proved to be non-toxic and were used in subsequent experiments. The effect of cordycepin on $\alpha$-MSH- and IBMX-induced cellular melanin synthesis and tyrosinase activity were investigated. Tyrosinase was chosen for study, given its importance during melanin synthesis; suppression of melanin production is always dependent on the inhibition of tyrosinase activity. The effect of cordycepin on $\alpha$-MSH- and IBMX-stimulated melanin synthesis is summarized in Fig. 1A and B. Both extracellular and intracellular melanin content in cordycepintreated cells decreased significantly in a dose-dependent fashion, while it markedly increasingly compared to control cells in cells treated with $\alpha$-MSH or IBMX alone. The effects of cordycepin on $\alpha$-MSH- and IBMX-induced tyrosinase activity are shown in Fig. 1C. In a cell-based tyrosinase assay, cordycepin inhibited tyrosinase activity in a dose-dependent manner. Arbutin was used as positive control; it significantly inhibited extracellular and intracellular melanin content and tyrosinase activity in $\mathrm{B} 16 \mathrm{~F} 10$ cells at $200 \mu \mathrm{M}$. These results are consistent with the idea that reduced production of melanin is due to the suppression of tyrosinase activity.

In order to evaluate the mechanism(s) of the inhibitory effect of cordycepin on cellular melanin synthesis, the protein expression levels of melanogenic-related proteins such as tyrosinase, TRP1 and TRP2 were assessed by Western blotting. The expression of tyrosinase, TRP1 and TRP2 were decreased in $\alpha$-MSH- and IBMX-treated melanoma cells in the presence of cordycepin, as compared to control cells (Fig. 2A). In addition, the results of real-time RT-PCR of $\alpha-\mathrm{MSH}-$ and 
IBMX-treated B16F10 melanoma cells treated with cordycepin confirmed that cordycepin could also inhibit the mRNA level of tyrosinase, TRP1 and TRP2 (Fig. 2B).

MITF is a transcription factor that regulates melanogenic genes such as Tyr, Trp1 and Trp2. The promoter region of the MITF gene contains binding sites for transcription factors including SOX10, PAX3, TCF/LEF and CREB $(28,29)$. The expression of MITF could be increased by the phosphorylation of CREB. CREB phosphorylation induced by $\alpha$-MSH and IBMX was decreased by cordycepin (Fig. 3). Taken together, the results are consistent with the suggestion that the inhibitory effect of cordycepin on the down-regulation of the expression of MITF can be attributed to its inhibition of CREB phosphorylation.

MITF is a specific transcription factor that is involved in the regulation of melanocyte differentiation, pigmentation, proliferation, and survival. MAPKs are play an important role in the control of melanogenesis in melanocytes, and the expression of MITF is activated by p38-MAPK pathway. Increased expression of MITF ultimately increases melanin synthesis, whereas, the ERK and Akt pathways are down-regulated. In our study, both p-ERK and p-Akt were increased by cordycepin. Furthermore, bloking of p-ERK and p-AKT increased the expression of TPR-1, TRP2 and melanin contents. These results also demonstrate that the inhibitory effect of cordycepin is dependent on the ERK and AKT signaling pathways (Fig. 4).

In this study, we investigated whether cordycepin inhibits melanin synthesis in $\alpha$-MSH- and IBMX-induced melanocytes by suppressing expression of MITF, tyrosinase and TRP-1. On the basis of our results, we suggest that cordycepin is a potent hypopigmenting agent with implications in various dermatologic hyperpigmentation disorders, such as freckles and melasma, and has beneficial effects in whitening cosmetics.

\section{References}

1. Sato K, Morita M, Ichikawa C, Takahashi $\mathrm{H}$ and Toriyama M: Depigmenting mechanisms of all-trans retinoic acid and retinol on B16 melanoma cells. Biosci Biotechnol Biochem 72: 2589-2597, 2008.

2. Lee YS, Kim HK, Lee KJ, Jeon HW, Cui S, Lee YM, Moon BJ, Kim YH and Lee YS: Inhibitory effect of glyceollin isolated from soybean against melanogenesis in B16 melanoma cells. BMB Rep 43: 461-467, 2010.

3. Chung SY, Seo YK, Park JM, Seo MJ, Park JK, Kim JW and Park CS: Fermented rice bran downregulates MITF expression and leads to inhibition of $\alpha-\mathrm{MSH}$-induced melanogenesis in B16F1 melanoma. Biosci Biotechnol Biochem 73: 1704-1710, 2009.

4. Ando H, Kondoh H, Ichihashi M and Hearing VJ: Approaches to identify inhibitors of melanin biosynthesis via the quality control of tyrosinase. J Invest Dermatol 127: 751-761, 2007.

5. Shimoda N, Mutou Y, Shimura N, Tsukimoto M, Awaya A and Kojima S: Effect of heterocyclic pyrimidine compounds on UVB-induced cell damage in human keratinocytes and on melanogenesis in mouse B16 cells. Biol Pharm Bull 33: 862-868, 2010

6. Sato K, Takahashi H, Iraha R and Toriyama M: Down-regulation of tyrosinase expression by acetylsalicylic acid in murine B16 melanoma. Biol Pharm Bull 31: 33-37, 2008.

7. Hasegawa K, Furuya R, Mizuno H, Umishio K, Suetsugu M and Sato K: Inhibitory effect of Elephantopus mollis H.B. and $\mathrm{K}$. extract on melanogenesis in B16 murine melanoma cells by downregulating microphthalmia-associated transcription factor expression. Biosci Biotechnol Biochem 74: 1908-1912, 2010.

8. Widlund HR and Fisher DE: Microphthalamia-associated transcription factor: a critical regulator of pigment cell development and survival. Oncogene 22: 3035-3041, 2003.
9. Osawa M: Melanocyte stem cells. Stem Book. Harvard Stem Cell Institute, Cambridge, 2009.

10. Jiang Z, Xu J, Long M, Tu Z, Yang G and He G: 2, 3, 5 , 4'-Tetrahydroxystilbene-2-O-beta-D-glucoside (THSG) induces melanogenesis in B16 cells by MAP kinase activation and tyrosinase upregulation. Life Sci 85: 345-350, 2009.

11. Ye Y, Chu JH, Wang H, Xu H, Chou GX, Leung AK, Fong WF and $\mathrm{Yu}$ ZL: Involvement of p38 MAPK signaling pathway in the anti-melanogenic effect of San-bai-tang, a Chinese herbal formula, in B16 cells. J Ethnopharmacol 132: 533-535, 2010.

12. Singh SK, Sarkar C, Mallick S, Saha B, Bera R and Bhadra R: Human placental lipid induces melanogenesis through p38 MAPK in B16F10 mouse melanoma. Pigment Cell Res 18: 113-121, 2005.

13. Hirata N, Naruto S, Ohguchi K, Akao Y, Nozawa Y, Iinuma M and Matsuda $\mathrm{H}$ : Mechanism of the melanogenesis stimulation activity of (-)-cubebin in murine B16 melanoma cells. Bioorg Med Chem 15: 4897-4902, 2007.

14. Jang JY, Lee JH, Kang BW, Chung KT, Choi YH and Choi BT: Dichloromethane fraction of Cimicifuga heracleifolia decreases the level of melanin synthesis by activating the ERK or AKT signaling pathway in B16F10 cells. Exp Dermatol 18: 232-237, 2009.

15. Kim S, Lee J, Jung E, Lee J, Huh S, Hwang H, Kim Y and Park D: 6-Benzylaminopurine stimulates melanogenesis via cAMPindependent activation of protein kinase A. Arch Dermatol Res 301: 253-258, 2009.

16. Lei TC,Zhu WY,Xia MY,Zhang MH and Fan WX: Comparative studies on regulation of melanogenesis in the responese of murine melanoma cells to ammonium glycyrrhizinate, arbutin and hydroquinone. J Clin Dermatol 29: 69-72, 2000.

17. Chang MS, Choi MJ, Park SY and Park SK: Inhibitory effects of Hoelen extract on melanogenesis in B16/F1 melanoma cells. Phytother Res 24: 1359-1364, 2010.

18. Villareal MO, Han J, Yamada P, Shigemori $\mathrm{H}$ and Isoda $\mathrm{H}$ : Hirseins inhibit melanogenesis by regulating the gene expressions of Mitf and melanogenesis enzymes. Exp Dermatol 19: 450-457, 2010.

19. Yoon WJ, Kim MJ, Moon JY Kang HJ, Kim GO, Lee NH and Hyun CG: Effect of palmitoleic acid on melanogenic protein expression in murine b16 melanoma. J Oleo Sci 59: 315-319, 2010.

20. Noh EM, Kim JS, Hur H, Park BH, Song EK, Han MK, Kwon KB, Yoo WH, Shim IK, Lee SJ, Youn HJ and Lee YR: Cordycepin inhibits IL-1b-induced MMP-1 and MMP-3 expression in rheumatoid arthritis synovial fibroblasts. Rheumatology (Oxford) 48: 45-48, 2009.

21. Kim HG, Shrestha B, Lim SY, Yoon DH, Chang WC, Shin DJ, Han SK, Park SM, Park JH, Park HI, Sung JM, Jang Y, Chung N, Hwang KC and Kim TW: Cordycepin inhibits lipopolysaccharide-induced inflammation by the suppression of NF-kappa B through Akt and p38 inhibition in RAW 264.7 macrophage cells. Eur J Pharmacol 545: 192-199, 2006.

22. Shin S, Lee S, Kwon J, Moon S, Lee S, Lee CK, Cho K, Ha NJ and Kim K: Cordycepin suppresses expression of diabetes regulating genes by inhibition of lipopolysaccharide-induced inflammation in macrophages. Immune Netw 9: 98-105, 2009.

23. Shin S, Moon S, Park Y, Kwon J, Lee S, Lee CK, Cho K, Ha NJ and Kim K: Role of cordycepin and adenosine on the phenotypic switch of macrophages via induced anti-inflammatory cytokines. Immune Netw 9: 255-264, 2009.

24. Jeong WJ, Jin CY, Kim GY, Lee JD, Park C, Kim GD, Kim WJ, Jung WK, Seo SK, Choi IW and Choi YH: Anti-inflammatory effects of cordycepin via suppression of inflammatory mediators in BV2 microglial cells. Int Immunopharmacol 10: 1580-1586, 2010.

25. Won SY and Park EH: Anti-inflammatory and related pharmacological activities of cultured mycelia and fruiting bodies of Cordyceps militaris. J Ethnopharmacol 96: 555-561, 2005.

26. Nakamura K, Yoshikawa N, Yamaguchi Y, Kagota S, Shinozuka K and Kunitomo M: Antitumor effect of cordycepin (3'-deoxyadenosine) on mouse melanoma and lung carcinoma cells involves adenosine A3 receptor stimulation. Anticancer Res 26: 43-47, 2006.

27. Fuller BB, Lunsford JB and Iman S: Alpha-melanocyte-stimulating hormone regulation of tyrosinase in Cloudman S-91 mouse melanoma cell cultures. J Biol Chem 262: 4024-4033, 1987.

28. Bellei B, Flori E, Izzo E, Maresca V and Picardo M: GSK3 $\beta$ inhibition promotes melanogenesis in mouse B16 melanoma cells and normal human melanocytes. Cell Signal 20: 1750-1761, 2008.

29. Kim DS, Jeong YM, Park IK, Hahn HG, Lee HK, Kwon SB, Jeong JH, Yang SJ, Sohn UD and Park KC: A new 2-imino1,3-thiazoline derivative, KHG22394, inhibits melanin synthesis in mouse B16. Biol Pharm Bull 30: 180-183, 2007. 